\title{
Ossifying fibroma of mandible-A concise radiographic exploration
}

\author{
Preethi Poonja ${ }^{1}$, Atul Sattur ${ }^{2}$, Krishna Burde ${ }^{2}$, Kirthy Nandimath ${ }^{2}$, Kaveri Hallikeri ${ }^{2}$ \\ (1) AJ Institute Of Dental Sciences, Oral Medicine And Radiology, Karnataka, India \\ (2) SDM College Of Dental Sciences, Oral Medicine And Radiology, Karnataka, India
}

Date submitted:

Jan 31, 2019

Date accepted:

Feb 26, 2019

Online publication date:

September 15, 2019

\section{Corresponding Author:}

Preethi Poonja

AJ Institute of Dental Sciences,

Oral Medicine And Radiology,

Karnataka, India

preetipoonja@gmail.com

\begin{abstract}
Ossifying fibroma (OF) is a rare benign fibro-osseous lesion that occurs in the mandible more frequently than the maxilla. It is usually diagnosed in the third to fourth decades of age, with a female predilection. This case report describes an asymptomatic bony swelling of the mandible in a 21 year old female patient which gradually progresses to a remarkable size causing discomfort. The present case provides comprehensive radiological explorations that include conventional as well as the 3D imaging combined with histopathological investigations that confirmed the diagnosis of Ossifying fibroma. The lesion was completely excised with satisfactory healing.
\end{abstract}

Keywords: fibro-osseous lesions, 3D imaging, bony tumors, jaw swellings.

\section{Introduction}

Fibro-osseous lesions are characterized by the presence of bone marrow that has changed into fibrous tissue and that contains mineralized material of varying appearances. Depending on the proportions of soft and mineralized tissue, they may be predominantly radiolucent, mixed radio-dense-radiolucent, or mainly radiodense (1). The case report highlights the importance of some fundamental radiographic features obtained from conventional radiographs along with the advanced imaging in confirming the diagnosis for bony lesions.

\section{Presentation of Case}

A 21 year old healthy female patient complains of swelling in the right lower front region of the jaw since 3 months. Patient gives a history of gradual increase in the size of the swelling. The swelling has been causing discomfort to the patient and hence she reported to the department of oral medicine and radiology. The patient does not give history of trauma in the region and the medical history is non-contributory.

On Extra oral examination the swelling was not causing any facial asymmetry. Lymph nodes were non tender and not palpable. Lips were competent. Intraorally the lesion was well defined that extended inferiorly from the vestibular area of the canine and premolars on the right side of the mandible. The size of the lesion was around $1 \times 1 \mathrm{~cm}$ in diameter. The mucosa over the lesion appeared normal with no change in the surrounding mucosa (Figure 1a). On percussion of $41,42,43,44$ no tenderness was present. 43 was displaced lingually with no mobility. On palpation the swelling appeared firm and bony in consistency and was non tender with no discharge from the area.

A provisional diagnosis of benign fibro-osseous lesion was given. A differential diagnosis of Adenamatoid odontogenic tumor was also considered.

Orthopantamograph (OPG) showed a well-defined unilocular mixed lesion with a ground-glass appearance internally surrounded by a thin radiopaque margin extending from the distal aspect of canine to the mesial aspect of the premolar causing displacement of teeth. Root resorption is not evident (figure 1b).

Digital volumetric tomography (DVT) revealed expansion of the buccal cortical plate which was intact and thinning of lingual cortical plate with radiopaque calcified specks within the inter- 


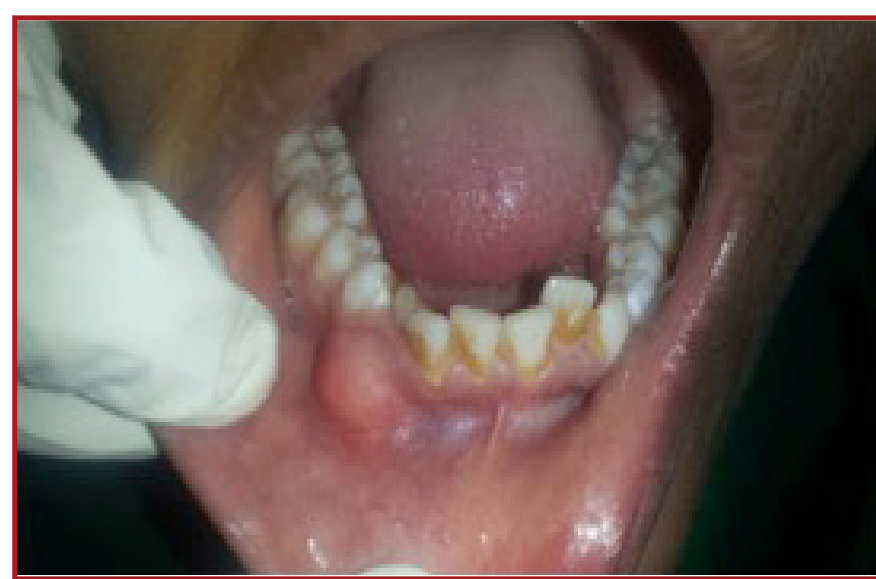

Figure 1a. Clinical image shows a well defined solitary bony swelling in the right mandible in relation to $42,43,44$ region.

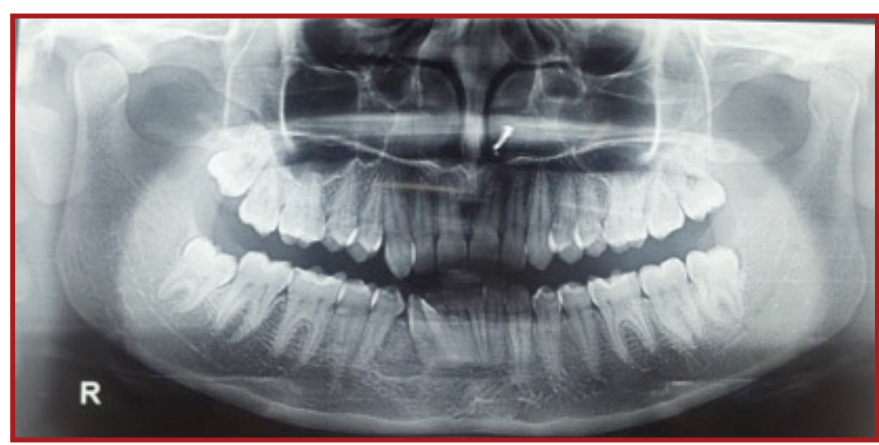

Figure 1b. Orthopantamography reveals a well defined unilocular mixed lesion with a well-corticated margin in the periapical region causing displacement of roots in relation to 43 and 44 .

nal structure in the axial view. A 3D Recon view also revealed mixed density due to a variable amount of radiopaque material between 43 and 44 with displacement of roots. Radiographic diagnosis of ossifying fibroma was established and the patient was subjected to excisional biopsy of the lesion (figure 2a).

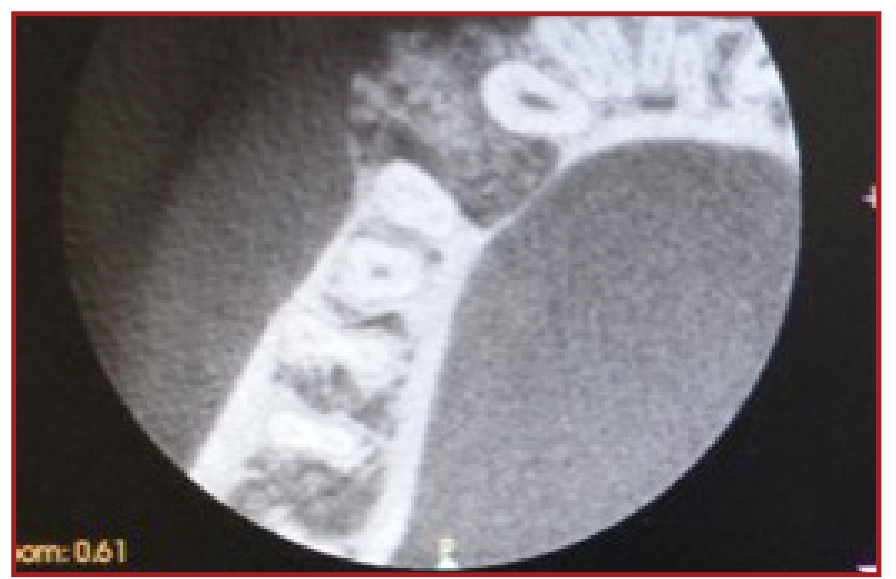

Figure 2a. Axial view of DVT shows expansion Of the buccal cortical plate and thinning of the Lingual cortical plate with flecks of radiopaque material within the internal structure.

The histopathological features showed interconnected mature bony trabeculae with fibrous stroma that confirmed the diagnosis of Ossifying fibroma (figure $2 b$ ). The patient was followed up over a phone call since she was from a different city and was unable to travel due to financial constrains. The patient was satisfied with the treatment and had no fresh complains.

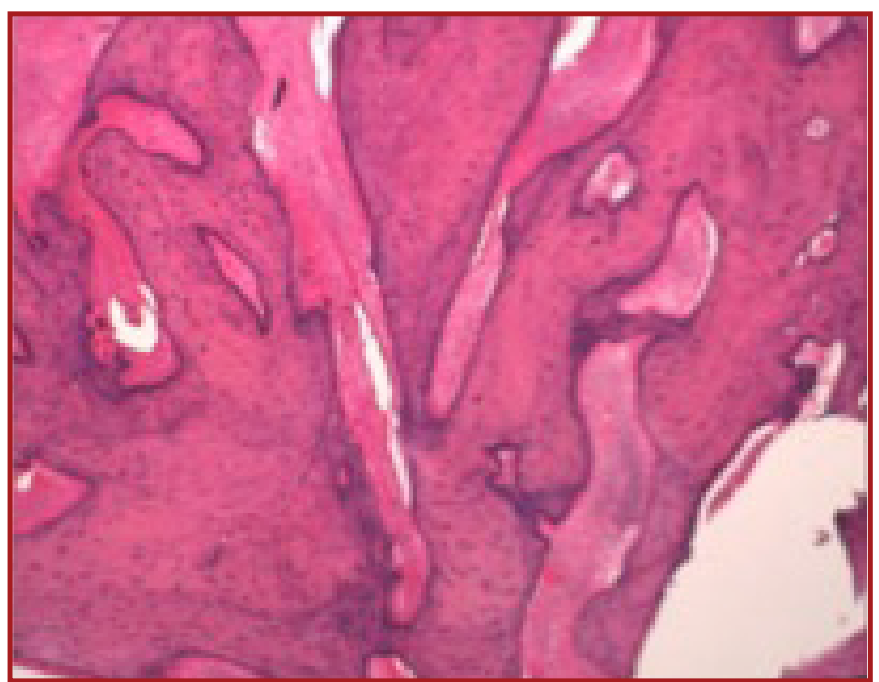

Figure $2 \mathrm{~b}$. Photomicrograph shows $10 \mathrm{x}$ view of interconnected mature bony trabeculae with fibrous stroma (H\&E).

\section{Discussion}

Ossifying fibroma (OF) of the jaw is a slow-growing benign tumor with origins in the periodontal ligament. The lesion is mainly seen in the mandible and shows more affinity to the molar and premolar region. Although it can affect patients at all ages, its peak incidence is in the 20- to 40-year age group and shows a predilection for women. In most cases, a single, well-demarcated lesion is diagnosed. In the present case the patient was a 21year old female and the lesion was present between the premolar and canine. The lesion had caused the divergence of the involved teeth without any evident root resorption. The lesion was asymptomatic and was progressing gradually over a period of 3 months without any facial asymmetry extraorally.

Based on clinical, 3D radiologic and histopathological findings, it is feasible to distinguish ossifying fibroma from other lesions which have clinical resemblances like fibrous dysplasia, hyperparathyroidism, osteitis deformans and diffuse sclerosing osteomyelitis and AOT (2).

It is important to stress that the COF is a sharply demarcated lesion. The hard tissues of the tumor do not fuse with the surrounding bone, except occasionally in limited areas. This is a significant feature in distinguishing a COF from a fibrous dysplasia, which usually blends with the surrounding bone (3).

Radiologically one distinguishing feature that may be present is a soft tissue capsule at the periphery not seen in fibrous dysplasia. OF can be distinguished from the previously mentioned bone dysplasias by its tumor like behavior. This is reflected in the growth of the lesion, which tends to be concentric within the medullary part of the bone with outward expansion approximately equal in all directions. Displacement of teeth is common in both but in OF it is from a specific point or epicenter (4).

Radiologically, cemento-ossifying fibroma shows a number of patterns depending on the degree of mineralization of the lesion. Eversole and colleagues (1985) studied 43 cases of ossifying fibroma in which radiographs were available and found 6 variations (5).

Ram.et.al presented a case of a 35 year old female patient with a swelling on the lower left jaw since 2 months on the body of mandible and extended along the inferior border of the mandible up to the submandibular region. The overlying mucosa was normal in appearance. 35, 36 and 37 were mobile and 
tender on percussion (6). In our case the patient was asymptomatic and the bony swelling was present in the region of 43 and 44 with no tenderness and mobility, but tooth displacement was evident.

\section{Conclusion}

Fibro-osseous lesions resemble variety of odontogenic and non odontogenic tumors. It is important for clinicians to have a broader perspectives of jaw swellings and consider them in the differentials. Thus definitive diagnosis requires an extensive understanding of the clinical, radiological and histopathological features of the bony lesions for proper treatment and management and to avoid recurrences.

Acknowledgement:The patient was evaluated by PP, KN, AS and KB. Data were collected and analyzed by PP, AS and KB. Manuscript was prepared by PP. Histopathology confirmation and photomicrographs were provided by $\mathrm{KH}$. All the authors have participated in the drafting of the manuscript.

Conflicts of Interests: The authors declared they do not have anything to disclose regarding conflict of interest with respect to this manuscript.

\section{References}

1. Slootweg PJ. Maxillofacial fibro-osseous lesions: classification and differential diagnosis. Semin Diagn Pathol. 1996 May; 13 (2): 104-112.

2. Brügger OE, Reichart PA, Werder P, Altermatt HJ, Bornstein MM. Asymptomatic ossifying fibroma of the mandible: a case presentation. Quintessence Int. 2012; 43: 381-385

3. Reichart, P.A., Philipsen, H.P., Eds. Odontogenic Tumors and Allied Lesions Quintessence Publishing, London, 2004:273-279.

4. White SC, Pharoah MJ. Diseases of bone manifested in jaws. Oral Radiology: Principle and Interpretation. 6th ed. New Delhi: Mosby; 2004:500-501.

5. Langlais RP, Langland OE, Notje CJ. Diagnostic Imaging of the Jaws. 1st ed. USA Baltimore: Williams \& Wilkins; 1995: 551-552.

6. Ram R, Singhal A, Singhal P. Cemento-ossifying fibroma. Contemp Clin Dent. 2012; 3(1): 83-85. 